or spindle formation even though they enter and presumably complete $S$ phase. The absence of these characteristic mitotic events points to the existence of a checkpoint mechanism that makes the activation of the M-phase controlling protein kinase $\mathrm{p} 34^{c d c 2}$ (reviewed in ref. 11) tightly dependent upon the successful duplication of the centrosome. The question then becomes how the coordination of centrosome duplication and kinase activation might be achieved. Whatever the molecular mechanisms at work, they could require direct interaction of the mitotic kinase itself with centrosomes, as outlined both by immunofluorescence studies in mammalian $^{12,13}$, insect (D. M. Glover, personal communication) and yeast ${ }^{14}$ cells and by cell fractionation experiments ${ }^{12}$. Accordingly, the absence of such an interaction in centriole-free karyoplasts would preclude the full activation of the protein kinase.

A striking property of the centrosome is that its duplication cycle encompasses all four phases of the cell cycle. In this context one might wonder why centrosome duplication starts so early in the cell cycle. A conceptually appealing hypothesis is that the discrete events of this process define a sequence of checkpoints: the failure of any one of these events would affect progression of the cell cycle by a feedback mechanism. In other words, the centrosome cycle might provide the cell with a kind of logical cog that monitors time and mass increase, thereby contributing to the coupling of cell growth and cell division.

Eric Bailly and Michel Bornens are in the CNRS Centre de Genetique Moleculaire, 91198 Gif-sur-Yvette Cedex, France.

\title{
Stalking the magma ocean
}

\section{David J. Stevenson}

PLANETS and smaller bodies are evidently differentiated, at least into a metallic core, silicate mantle and buoyant crust. Much of the differentiation occurred or began when the body was much hotter than now, back at the time of planetary formation around 4.5 thousand million years ago. It has been popular for some time to invoke a 'magma ocean' epoch when the temperatures were so high that most of the body was molten. During and immediately after this period, differentiation is thought to have been quick because of the rapidity of relative motion of silicate crystals or dense iron droplets relative to the low-viscosity magma. Recent attention has been focused on the Earth's putative magma ocean, and geologists assembled at a workshop in December* to debate evidence for and against such oceans on Earth and other bodies. This gathering showed a growing strength of opinion and argument in favour of their existence, but also a growing awareness of the lack of compelling observational evidence. It could be that the terrestrial magma ocean is merely a convenient metaphor for a complicated epoch in which the Earth's mantle can be treated neither as mostly fluid nor as mostly solid. This two-phase or multiphase problem still defies our geochemical and fluid dynamical understanding.

Historically, magma oceans were first invoked for the Moon. In the Apollo days, John Wood ${ }^{1}$ and others developed a simple but still very successful picture

* Workshop on the Physics and Chemistry of Magma Oceans from 1 bar to 4 Mbar, Burlingame, California, 6-8 December 1991 for the origin of lunar highland crust from fractional crystallization of the lunar magma ocean. At the workshop, Paul Warren (Univ. California, Los Angeles) and others discussed further tests of the lunar ocean concept, which seems to be holding up well despite persisting uncertainties about how it works geophysically. Jeff Taylor (Univ. Hawaii) discussed evidence for magma oceans on asteroids, mainly through consideration of meteorites; here, too, the evidence is good although the heat source for extensive melting remains mysterious.

These extraterrestrial examples are placid compared to the stormy violence of the proposed terrestrial magma ocean, in which convective velocities may have been of the order of a metre per second. This is the area where the workshop was most lively, and where the tension between geochemical and geophysical perspectives has previously been most evident. Rama Murthy (Univ. Minnesota) presented his provocative argument ${ }^{2}$ for very high temperatures of metal-silicate fractionation during core formation, supportive of a magma ocean. His argument is that the abundances of siderophile (iron-loving) elements in the present mantle can be understood in terms of equilibrium partitioning at $3,000-3,500^{\circ} \mathrm{C}$. This met considerable opposition, most notably from Mike Drake (Univ. Arizona) and John Jones (Johnson Space Center, Houston), who argue that a more careful assessment of the chemistry of this equilibration is required. Nevertheless, Murthy has forced us to shift attention to the possible consequences of very-hightemperature geochemistry.

Carl Agee (Harvard Univ.) presented his interesting experiments on the highpressure melting of carbonaceous chondrites (primitive meteoritic material), and argued that the mantle we know most about (the peridotitic upper mantle) is a differentiate of the magma ocean epoch, with a higher $\mathrm{Mg} / \mathrm{Si}$ ratio than the bulk Earth. The lower mantle may also be relatively iron-rich, as some have argued on different geophysical grounds, although this is a less certain prediction because of the unknown extent to which the core may have incorporated $\mathrm{FeO}$ rich material from the lowermost mantle. However, Agee admits that Earth is not an assembly of carbonaceous chondrites, and more relevant experiments need to be performed.

Ted Ringwood (Australian National Univ.) and his colleagues, although not present at the workshop, have challenged the magma ocean concept by pointing out that the partitioning of trace elements (notably some rare earths) between perovskite and coexisting melt leads to a differentiation that would violate the nearly chondritic pattern of these particular elements in the upper mantle ${ }^{3}$. Geophysicists have risen to this challenge, and calculations presented by Yutaka Abe (Nagoya Univ.) and Viatcheslav Solomatov (California Inst. Technol.) and myself show that the separation of liquid from melt need not occur (as first pointed out by Tonks and Melosh ${ }^{4}$, at least until the crystal fraction reaches the value at which a solid matrix begins to develop.

There are hints in these calculations that differentiation does nevertheless occur in the later crystal-rich phase, that it is not of the kind feared by Ringwood and colleagues, but that it may indeed lead to a layered mantle with major element $(\mathrm{Mg} / \mathrm{Si}, \mathrm{Mg} / \mathrm{Fe})$ contrasts, as Agee argues. This brings us back to the longstanding, still unresolved debate on whether the mantle is compositionally layered (a debate that is often, perhaps erroneously, described as layered versus whole-mantle convection). The exciting challenge that remains is to relate our developing understanding of early Earth with our current views of mantle convection, geochemistry and core structure. $\square$

David J. Stevenson is in the Division of Geological and Planetary Sciences, California Institute of Technology, Pasadena, California 91125, USA. 1. Wood, J. et al. Proc. 1st Lunar Science Conf. 965-988
(1970).

2. Murthy, V. R. Science 253, 303-305 (1991)

3. Ringwood, A. E. in Origin of the Earth (eds Newsom, H. E. \& Jones, J. E.) 101-134 (Oxford University Press, 1990).

4. Tonks, W. B. \& Melosh, H. J. Origin of the Earth (eds Newsom, H. E. \& Jones, J. E.) 101-134 (Oxford University Press, 1990). 